\title{
DUELO PROLONGADO Y FACTORES ASOCIADOS
}

\section{PROLONGED GRIEF AND RELATED FACTORS}

\author{
Patricia Estevan Burdeus', Cristina De Miguel Sánchez², Roberto Álvarez Álvarez³, Esther \\ Martín Molpeceres ${ }^{4}$, Borja Múgica Aguirre ${ }^{5}$, Ana Riestra Fernández², Aránzazu Gutiérrez \\ Ruiz², Lara Sanz Ventureira², Fernando Vicente Sánchez ${ }^{2}$, Gema García Jiménez², Fran- \\ cisco Javier García Oliva', Rhut Cristóbal Saenz de Manjares', Amparo Corral Rubio', \\ Vanesa Bonivento Martínez' , José Antonio Guechoum González', María Carrera Lavín', \\ María Jesús López Rodríguez ${ }^{1}$ Cristina Morán Tiesta ${ }^{6}$ e Isabel Del Cura González \\ ' Centro de Salud de Lavapiés. Gerencia de Atención Primaria, Madrid. \\ ${ }^{2}$ Equipo de Soporte de Atención Domiciliaria Paliativa de Espronceda. Gerencia de Atención Primaria, \\ Madrid. \\ ${ }^{3}$ Unidad de Cuidados Paliativos - Daño Cerebral. Fundación Instituto San José, Madrid. \\ ${ }^{4}$ Unidad de Cuidados Paliativos, Fundación Jiménez Díaz, Madrid \\ ${ }^{5}$ Unidad de Cuidados Paliativos, Centro de Cuidados Laguna, Madrid. \\ 6. Centro de Salud Lucero. Gerencia de Atención Primaria, Madrid. \\ 7. Unidad de Apoyo a la Investigación, Gerencia de Atención Primaria. Servicio Madrileño de Salud, Madrid
}

Resumen

Objetivo: Describir factores asociados a la presencia de Duelo Prolongado (DP) medido con el cuestionario Prolonged Grief Disorder-13 (PG-13) en pacientes que han perdido a un ser querido entre 6 y 18 meses.

Método: Estudio transversal. Dolientes mayores de edad procedentes de atención primaria, hospitales de cuidados paliativos y un equipo de soporte a la atención domiciliaria paliativa en Madrid. Se recogieron variables del fallecido y del doliente: sociodemográficas, socioeconómicas, antecedentes psiquiátricos, utilización de recursos sanitarios, Trastorno por Ansiedad Generalizada (TAG), Trastorno Depresivo Mayor (TDM), apoyo social, percibido y duelo prolongado con PG-13 y el Inventario Texas Revisado de Duelo (ITRD). Se calcularon medidas de tendencia central y distribución de frecuencias, se calculo asociación entre variables con $\mathbf{c}^{2}$ y t-student, se ajustó modelo multivariante.

\section{Abstract}

Objective: To describe associated factors with the presence of Prolonged Grief Disorder (PGD) diagnosed by the Prolonged Grief Disorder-13 (PG-13) questionnaire between 6 and 18 months after the loss of a loved one.

Method: Cross-sectional study. Adults chosen from a sample coming from primarycare services, hospices, and a home care team, all of them in Madrid.

Variables from the deceased and the bereaved were collected: demographic, socioeconomic, psychiatric records, medical visits, Generalized Anxiety Disorder (GAD), Major Depressive Disorder (MDD), social perceived support and PGD diagnosed by PG-13 and Texas Revised Inventory of Grief (TRIG). Multivariate linear regression analysis was used to identify factors associated with PGD.

Results: Prevalence of PGD was $7.023 \%$. PGD was associated with female gender,

\section{Correspondencia:}

Patricia Estevan Burdeus

Avda. Ciudad de Barcelona 43,5 F. 28007. Madrid.

E-mail: patricia.estevan@salud.madrid.org
Proyecto financiado con Fondos de Investigación en Salud del ISCIII (PI12/00952) "Validación y adaptación trascultural del cuestionario Prolonged Grief Disorder-13 (PG-13) para la detección precoz de duelo prolongado" y con la Beca "Isabel Fernández" de la Sociedad Española de Medicina de Familiar y Comunitaria para la realización de tesis doctorales en la edición 2012. 
Resultados: La prevalencia de DP fue de $7,023 \%$. La presencia de DP se asoció significativamente con ser mujer, no tener trabajo, ingresos familiares bajos, fallecido más joven, enfermedad no susceptible de recibir cuidados paliativos, historia de ansiedad y/o depresión antes y después de la pérdida, haber consultado a un psiquiatra, haber tomado psicofármacos, una mayor frecuentación de los servicios de urgencias, medicina y enfermería de atención primaria, con TAG, EDM, apoyo social percibido bajo y DP según los criterios del ITRD. En el análisis multivariante se asoció con haber visitado al psiquiatra, EDM y con ingresos mensuales inferiores a 2.000 euros.

Conclusiones: Podemos modificar pocos de estos factores, pero conocerlos e identificar con prontitud a estos pacientes nos permitirá derivarlos a tratamientos apropiados como terapia específica de duelo.

Palabras clave: Duelo, duelo prolongado, factor de riesgo, modelos lineales. joblessness, low family incomes, a younger deceased, an illness not suitable for palliative care, anxiety or depression records before and after the loss, psychiatrist support, the use of psychotropic medication, increasing visits to emergency and primary care services, GAD, MDD, low social perceived support and the diagnosis of PGD using the TRIG.

Multivariate linear regression showed that PGD was associated with psychiatrist support, MDD and an income under 2000 euros.

Conclusions: Just a few of these risk factors can be modified by us (psychiatrist support, treatment of anxiety or depression) but by knowing them we will be able to have an early diagnosis and refer them to the appropriate resources like specific grief therapy as soon as possible.

Keywords: Grief, prolonged grief disorder, risk factor, linear models.

\section{INTRODUCCIÓN}

La pérdida de un ser querido es uno de los acontecimientos vitales más dolorosos al que inevitablemente se ha de enfrentar toda persona, y generalmente, en más de una ocasión a lo largo de su vida. El sufrimiento que causa una pérdida es muy intenso pero disminuye lentamente con el paso del tiempo y finalmente se restablecen el estado de ánimo y los niveles previos de actividad social y laboral.

Se calcula que un $5-20 \%$ de los afectados $^{(1)}$ padece durante meses y años los síntomas iniciales del duelo con la misma intensidad, lo que les provoca un deterioro, un aumento de la morbilidad ${ }^{(2)}$, mortalidad $^{(3)} y$ del riesgo de suicidio(4).

Debido a la falta de consenso en la denominación del duelo que no evoluciona satisfactoriamente se ha hablado según las épocas de duelo prolongado, patológico, traumático o complicado ${ }^{(5,6)}$. Para unificar la terminología de ahora en adelante utilizaremos el término de duelo prolongado siguiendo las recomendaciones más actuales ${ }^{(7)}$.

Entre los factores que definen el duelo prolongado se encuentran síntomas de ansiedad por separación, anhelo muy intenso por el fallecido, punzadas de dolor y pena muy profunda a diario durante más de seis meses. Se acompaña de síntomas cognitivos, conductuales y emocionales como evitar lugares, personas y cosas que recuerdan al fallecido, sentirse aturdido o paralizado, confundido acerca del papel en la vida, dificultad para aceptar la pérdida, para confiar en los demás, sentir amargura o sentirse bloqueado o pensar que le será imposible seguir adelante con su vida en el futuro y sentir que la vida no tiene sentido. Al menos 5 de estos síntomas deben presentarse con intensidad elevada o muy elevada y se deben acompañar de deterioro funcional significativo con una disminución notoria de las actividades en 
el ámbito doméstico, social o laboral durante 6 meses al menos tras la pérdida.

El duelo prolongado responde mejor a terapias específicas de duelo que a otras terapias ${ }^{(8)}$, los estudios con fármacos no son concluyentes, aunque parece que el escitalopram podría tener un papel prometedor ${ }^{(9)}$. La combinación de psicoterapia y tratamiento farmacológico parece que favorece la adhesión a la terapia y la probabilidad de respuesta al tratamiento en algunos estudios ${ }^{(10)}$, pero no en otros ${ }^{(11)}$, y en los casos positivos las muestras y el tamaño del efecto son pequeños.

Se han identificado distintos factores de riesgo de duelo prolongado. En cuanto al género, parentesco y causa de la muerte, se encuentra con más frecuencia en mujeres, cuando el fallecido es un hijo o la pareja y en aquellos cuya causa de muerte ha sido violenta, por accidente o por suicidio ${ }^{(12)}$. En relación a las circunstancias de la muerte y el entorno social, favorecen su aparición las situaciones de catástrofe en las que se unen pérdidas materiales y humanas múltiples, en mujeres que han perdido un hijo y no han conseguido tener otro, el número de hijos que sobreviven cuando uno fallece, la mala salud percibida, la falta de distracciones, el haber tenido que rescatar el cuerpo del familiar fallecido y no haber recibido apoyo psicológico de ningún tipo dificultan el proceso de duelo ${ }^{(13)}$. En la investigación de los factores personales que afectan a la evolución del duelo, con frecuencia los estudios son de pequeños tamaños y retrospectivos. La presencia de trastorno depresivo mayor, trastorno por estrés postraumático y diversos trastornos por ansiedad (trastorno de angustia con y $\sin$ agorafobia, agorafobia sin angustia, trastorno obsesivo-compulsivo, trastorno por ansiedad generalizada, trastorno por estrés postraumático o fobia social), han demostrado relacionarse con una peor evolución del duelo(14-16).
Se realizó este estudio cuyo objetivo fue determinar la prevalencia de duelo prolongado en la población con la que trabajamos, medida con los cuestionarios Prolonged Grief Disorder-13 (PG-13) ${ }^{(5)}$ y el Inventario Texas Revisado de Duelo (ITRD) $(17,18)$, describir el perfil de los dolientes con duelo prolongado y determinar la presencia de ansiedad, depresión y el apoyo social percibido.

\section{MATERIAL Y MÉTODOS}

Se diseñó un estudio descriptivo transversal, desarrollado en tres ámbitos: en atención primaria (Centros de Salud Lavapiés y Lucero), en hospitales de cuidados paliativos (Centro de Cuidados Laguna, Fundación Instituto San José y San Camilo) y en el Equipo de Soporte Atención Domiciliaria Paliativa (ESADP) de Espronceda de la Dirección Asistencial Centro, todos localizados en Madrid.

La población a la que se dirigió el estudio fueron mayores de 18 años que hubieran perdido un ser querido hacía más de 6 meses pero menos de 18 por cualquier causa en la comunidad de Madrid entre el 1 de mayo de 2014 y el 15 de mayo de 2015.

Los criterios de exclusión fueron, en el momento de inclusión en el estudio: demencia o deterioro cognitivo de moderado a severo, consumo de droga por vía parenteral, deficiencias sensoriales severas que impidieran la administración del cuestionario, trastorno mental grave (esquizofrenia o trastorno bipolar), cociente intelectual $<70$ o diagnóstico de retraso mental y antecedentes de intento autolítico o ideación suicida.

Los participantes se incluyeron por muestreo consecutivo y el tamaño de la muestra fue el resultante de los pacientes incluidos en el periodo de captación.

La captación de pacientes varió según el ámbito: en atención primaria el médico 
Figura 1. Diagrama de flujo del estudio

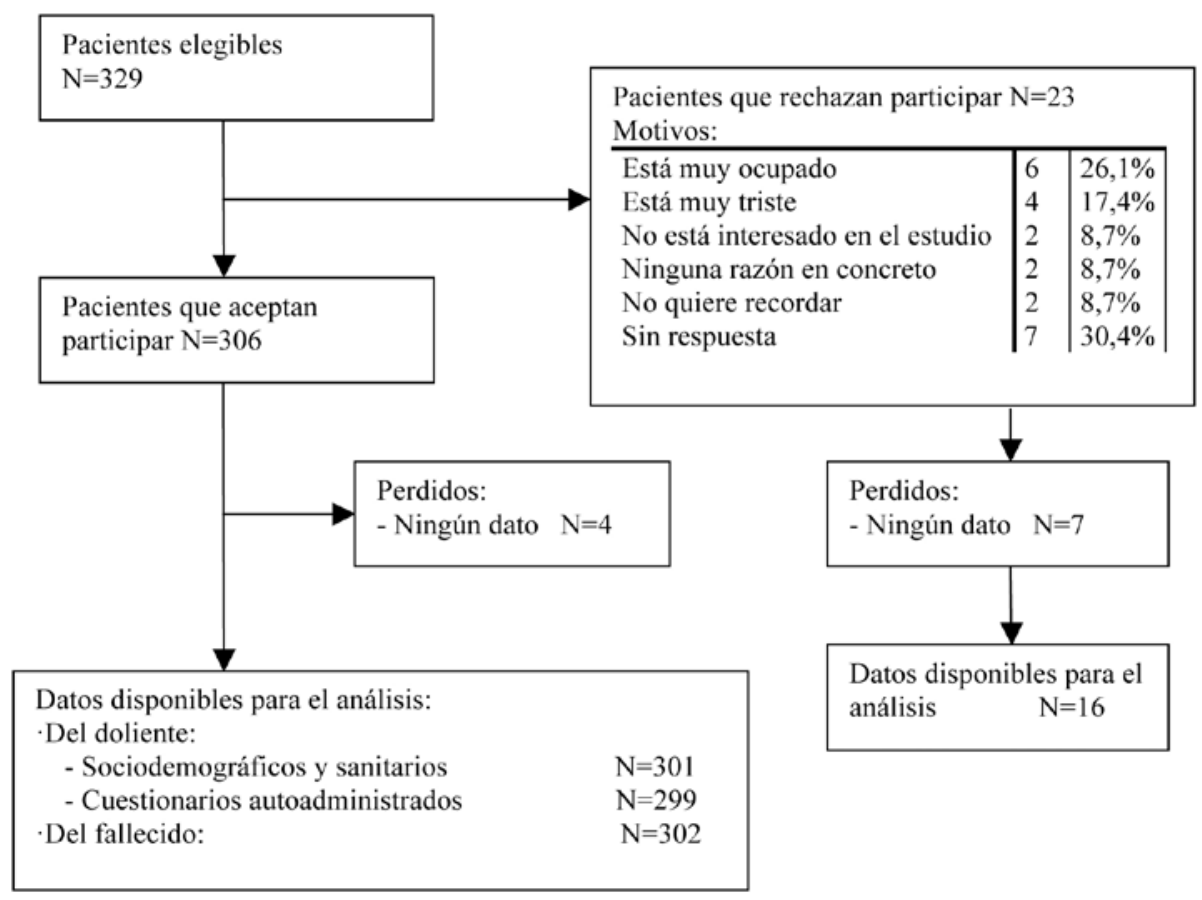

o la enfermera ofreció participar en consulta o vía telefónica a aquellos dolientes que cumplían los criterios de inclusión. Para contactar con los familiares de los pacientes fallecidos que habían sido atendidos por el ESADP se utilizaron los registros del servicio y se procedió a llamarles de manera consecutiva según la fecha de fallecimiento. Los psicólogos que trabajaban en hospitales de apoyo invitaron a participar a aquellos dolientes que estaban atendiendo en sus consultas en aquel momento y contactaron vía telefónica con aquellos que habían sido dados de alta por su parte pero que cumplían criterios de inclusión.

De aquellos dolientes que rechazaron participar se recogió el motivo de no participación y las variables sociodemográficas del doliente y del fallecido.

Los pacientes que aceptaron participar, firmaron el consentimiento informado y se les citó para realizar la entrevista. En ella se recogieron las variables del fa-
Ilecido y del doliente (sociodemográficas, socioeconómicas, antecedentes psiquiátricos, utilización de recursos sanitarios). Se utilizaron los criterios DSM-IV-R para diagnosticar Trastorno por Ansiedad Generalizada y Episodio Depresivo Mayor. Además cumplimentaron tres cuestionarios auto-administrados: el Cuestionario Duke $^{(19)}$ de apoyo social percibido que consta de 11 ítems con respuesta tipo Likert, del 1 al 5. El rango de puntuación está entre 11 y 55, una puntuación menor de 32 indica un apoyo social percibido bajo. Después el cuestionario Prolonged Grief Disorder-13 (PG-13) ${ }^{(5,20)}$, consta de 13 preguntas que se agrupan en 5 criterios, se consideró duelo prolongado cuando el doliente respondió afirmativamente a los criterios A, B, D y E y tuvo más de 4 puntos en 5 o más de las 9 cuestiones del criterio C. Y finalmente el Inventario Texas Revisado de Duelo ${ }^{(17,18)}$. El cuestionario consta de 21 ítems dividido en dos 


\section{Tabla 1. Variables sociodemográficas de los dolientes según la presencia de duelo prolongado medido por el cuestionario PG-13}

\begin{tabular}{|c|c|c|c|c|}
\hline & $\begin{array}{c}\text { Total } \\
(\mathrm{n}=299)\end{array}$ & $\begin{array}{c}\text { Duelo } \\
\text { Normal } \\
(n=278)\end{array}$ & $\begin{array}{c}\text { Duelo } \\
\text { Prolongado } \\
(\mathrm{n}=21)\end{array}$ & p-valor \\
\hline \multicolumn{5}{|l|}{ Sexo $(\%)$} \\
\hline - Mujeres & 70,9 & 69,4 & 90,5 & 0,04 \\
\hline - Hombres & 29,1 & 30,6 & 9,5 & \\
\hline Edad (años) Media (DE) & $53,5(17,2)$ & $53,5(17,1)$ & $56,1(14,7)$ & 0,35 \\
\hline \multicolumn{5}{|l|}{ Parentesco con fallecido (\%) } \\
\hline - Primer grado & 79,6 & 78,8 & 90,5 & 0,27 \\
\hline - Segundo, tercer grado y no familiar & 20,04 & 21,2 & 9,5 & \\
\hline $\begin{array}{l}\text { Tiempo transcurrido desde el } \\
\text { fallecimiento (meses) Media (DT) }\end{array}$ & $10,4(3,6)$ & $10,5(3,6)$ & $9,8(3,1)$ & 0,11 \\
\hline Número de hijos Media (DT) & $1,3(1,2)$ & $1,3(0,1)$ & $1,2(0,2)$ & 0,75 \\
\hline $\begin{array}{l}\text { Número de adultos en el domicilio } \\
\text { Media (DT) }\end{array}$ & $2,1(1,6)$ & $2,1(1,6)$ & $1,7(1,2)$ & 0,29 \\
\hline $\begin{array}{l}\text { Número de menores de } 14 \text { años en el } \\
\text { domicilio Media (DT) }\end{array}$ & $0,26(0,6)$ & $0,25(0,59)$ & $0,28(0,8)$ & 0,84 \\
\hline \multicolumn{5}{|l|}{ Nivel educativo (\%) } \\
\hline - Medio y alto & 75,1 & 75,9 & 61,9 & 0,15 \\
\hline - Bajo o analfabeto & 24,9 & 24,1 & 38,1 & \\
\hline \multicolumn{5}{|l|}{ Profesión/Ocupación (\%) } \\
\hline - Directores, técnicos y científicos & 42,5 & 43,9 & 23,8 & \\
\hline $\begin{array}{l}\text { - Profesionales de apoyo y empleados de } \\
\text { oficina }\end{array}$ & 18,4 & 17,6 & 28,6 & \\
\hline $\begin{array}{l}\text { - Empleados de los servicios de } \\
\text { restauración, vendedores y }\end{array}$ & & & & 0,22 \\
\hline explotaciones agropecuarias & 12,7 & 12,2 & 19 & \\
\hline $\begin{array}{l}\text { - Trabajadores cualificados de industrias } \\
\text { manufactureras, operarios de }\end{array}$ & & & & \\
\hline maquinaria y ocupaciones elementales & 26,4 & 26,3 & 28,6 & \\
\hline \multicolumn{5}{|l|}{ Situación Laboral (\%) } \\
\hline - Trabaja & 49,5 & 51,4 & 23,8 & 0,015 \\
\hline - No trabaja & 50,5 & 48,6 & 76,2 & \\
\hline $\begin{array}{l}\text { Ingresos mensuales familiares promedio } \\
\text { Media (DT) }\end{array}$ & $\begin{array}{l}2.149,03 \\
(1.196,4)\end{array}$ & $\begin{array}{l}2.211,4 \\
(1.199)\end{array}$ & $\begin{array}{l}1.436,8 \\
(903,3)\end{array}$ & $<0,01$ \\
\hline
\end{tabular}

partes, una de 8 y otra de 13 preguntas, con 5 respuestas tipo Likert. El resultado no es directo, es preciso pasarlo a una muestra de pacientes para calcular las puntuaciones medias de cada parte. En función de si la puntuación en una de las partes es mayor o menor a la media, se clasifica a cada doliente en cuatro grupos: Duelo Resuelto, Duelo inhibido, Duelo Retrasado y Duelo Prolongado. 
Para el análisis estadístico se realizó un análisis descriptivo de los sujetos, de las características sociodemográficas y de los diagnósticos mediante la determinación de las medidas de tendencia central y distribución de frecuencias, y se calcularon los IC para un $95 \%$. La asociación entre variables cualitativas se obtuvo mediante el test de la chi-cuadrado, y para comparar las medias de las variables cuantitativas se utilizó la prueba $t$ de student. Se establecieron comparaciones entre los dolientes con duelo normal y duelo prolongado en relación a las variables sociodemográficas, de prestación y utilización de servicios sanitarios y de nuevos diagnósticos mediante pruebas comparativas de medias y proporciones en grupos independientes. Se ajustó un modelo multivariante de regresión logística cuya variable dependiente fue la presencia de duelo prolongado medida con el cuestionario PG-13 y como variables independientes las variables que resultaron significativas en el análisis univariado y/o aquellas con criterio clínico. Para el análisis estadístico se utilizaron los programas SPSS versión 22.0 y Epidat 4.1.

Consideraciones éticas y legales: Se contempló la normativa española para el desarrollo de investigación biomédica. El estudio ha respetado los principios éticos básicos de autonomía, beneficencia, justicia y no maleficencia y su desarrollo siguió las normas de Buena Práctica Clínica, los principios enunciados en la Declaración de Helsinki (Fortaleza, Brasil, 2013), el Convenio de Oviedo (1997) y la Ley 41/2002, de 14 de noviembre, básica reguladora de la autonomía del paciente y de derechos y obligaciones en materia de información y documentación clínica (BOE 274 de 15 noviembre de 2002). Se respetaron las normas internacionales de protección de datos, así como la legislación española vigente (Ley Orgánica 15/1999 del 13/12/99 de Pro- tección de Datos de Carácter Personal, B.O.E. 298 de 14 noviembre de 1999).

El protocolo de este estudio fue autorizado por el Comité Ético de Investigación Clínica del Hospital Clínico San Carlos, y por la Comisión Central de Investigación de Atención Primaria de la Comunidad de Madrid.

El doliente se encuentra en una situación de vulnerabilidad extraordinaria, por eso además de seguir las normas generales de ética en la investigación se han seguido algunas recomendaciones emitidas en estudios realizados con dolientes ${ }^{(21)}$. Así, a aquellos que presentaron mayor estrés y malestar durante la entrevista, aún sin saber el resultado de los resultados de los cuestionarios se les ofreció la derivación a terapia específica de duelo sin coste alguno para ellos.

\section{RESULTADOS}

De los 329 pacientes elegibles rechazaron participar 23, el 6,9\%. En la figura 1 se presenta el diagrama de flujo del estudio. De los pacientes incluidos completaron el estudio 299, el 90,1\%. Solo se encontraron diferencias significativas entre la población de estudio y la población que rechazó participar en el número de personas con las que convivía, 2,3 en el grupo que aceptó participar frente a 0,8 personas $(p<0,01)$ en el grupo que rechazó y en el sexo del fallecido, mayoritariamente masculino, $87,5 \%$ frente al 53,6\%, $(p<0,05)$ en el grupo que aceptó participar.

Se encontró una prevalencia de duelo prolongado del 7,023\% (IC95\%: 4,4$10,05, p<0,001)$ en esta muestra.

En la tabla 1 se relacionan las principales características sociodemográficas de los dolientes en función de la presencia o no de duelo prolongado. La edad de los dolientes fue de media 53,5 (DT=17,2) años, en su mayoría mujeres, un 70,9\%, y parientes en primer grado del fallecido el $79,6 \%$. El tiempo trascurrido desde el fallecimien- 
to fue de 10,4 (DT=3,6) meses. El número medio de hijos fue de 1,28 (DT=1,25), el número de adultos en el domicilio fue de media 2,1 (DT=1,56), y el de menores de 14 años, $0,26(\mathrm{DT}=0,6)$. El nivel educativo fue bachiller o superior en el 75,1\%, y la categoría profesional principal recogida fue "Directores, técnicos y científicos" en un $42,5 \%$ de los dolientes. La situación laboral fue activo en un $49,5 \%$, con unos ingresos medios de 2.149,03 (DT=1.196,4) euros. La presencia de duelo prolongado se asoció con ser mujer, $90,5 \%$ frente a un $69,4 \%$ en el grupo de duelo normal, $\mathrm{p}<0,04$; no tener trabajo remunerado, $76,2 \%$ frente a $48,6 \%$, $\mathrm{p}<0,015 ; \mathrm{y}$ tener unos ingresos familiares promedio de 1.436,8 euros frente a los $2.421,4$ de los dolientes que presentaban duelo normal, $p<0,01$.
En la tabla 2 se muestran los datos sociosanitarios de los fallecidos de los dolientes del estudio. Los fallecidos fueron varones en un $57,1 \%$ de los casos, con una media de edad de 70,9 (DT=17,6) años. El 54,8\% fueron captados en el ESADP, seguido de los hospitales de cuidados paliativos, un $26,1 \%$. El fallecido presentó una enfermedad susceptible de recibir cuidados paliativos en el $72,6 \%$ de los casos, y los recibió en el $62,5 \%$ de los mismos. El lugar de fallecimiento más frecuente fue el domicilio en el 51,5\% de los casos. Una edad media menor del fallecido se asoció con la presencia de duelo prolongado, 60,6 (DT=21,8) años, frente a 71,6 (DT=17,1) años. El hecho de que el fallecido padeciera una enfermedad no susceptible de recibir cuidados

\section{Tabla 2. Variables sociodemográficas de los pacientes fallecidos según la presencia o no en dolientes de duelo prolongado medido por el cuestionario PG-13}

\begin{tabular}{|c|c|c|c|c|}
\hline & $\begin{array}{c}\text { Total } \\
(n=299)\end{array}$ & $\begin{array}{l}\text { Duelo Normal } \\
\qquad(\mathrm{n}=\mathbf{2 7 8 )}\end{array}$ & $\begin{array}{c}\text { Duelo } \\
\text { Prolongado } \\
(\mathrm{n}=21)\end{array}$ & p-valor \\
\hline \multicolumn{5}{|l|}{ Sexo $(\%)$} \\
\hline - Mujeres & 46,5 & 46,8 & 42,9 & \multirow[t]{2}{*}{0,7} \\
\hline - Hombres & 53,3 & 53,2 & 57,1 & \\
\hline Edad (años) Media (DT) & $70,9(17,6)$ & $71,6(17,1)$ & $60,6(21,8)$ & $<0,01$ \\
\hline \multicolumn{5}{|l|}{ Centro de captación (\%) } \\
\hline - Atención Primaria & 19,1 & 18,3 & 28,6 & \multirow{3}{*}{$<0,01$} \\
\hline - Equipo de paliativos a domicilio & 54,8 & 57,6 & 19 & \\
\hline - Hospitales de Cuidados Paliativos & 26,1 & 24,1 & 52,4 & \\
\hline \multicolumn{5}{|l|}{ Lugar de fallecimimiento $(\%)$} \\
\hline - Domicilio habitual & 51,5 & 52,2 & 42,1 & \multirow{4}{*}{0,018} \\
\hline - Hospital de Agudos & 31,6 & 32,4 & 21,1 & \\
\hline - Hospital de Crónicos & 13,1 & 12,6 & 21,1 & \\
\hline - Otros & 3,7 & 2,9 & 15,8 & \\
\hline \multicolumn{4}{|l|}{$\begin{array}{l}\text { Enfermedad susceptible de recibir } \\
\text { cuidados paliativos }(\%)\end{array}$} & \multirow{3}{*}{0,03} \\
\hline 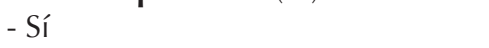 & 72,6 & 74,1 & 52,4 & \\
\hline - No & 27,4 & 25,9 & 47,6 & \\
\hline Recibió cuidados paliativos (\%) & 62,5 & 63,7 & 47,6 & 0,14 \\
\hline
\end{tabular}


paliativos se asoció con la presencia de duelo prolongado, un $47,6 \%$ frente a un $25,9 \%, p<0,03$, sin embargo el recibir o no cuidados paliativos no se relacionó con la presencia de duelo prolongado de manera estadísticamente significativa.

El $49,7 \%$ de los fallecimientos se produjeron por neoplasias, seguidos por enfermedades del aparato circulatorio, un 15,6\%, y del aparato respiratorio, un 11,6\%. El resto de las causas se muestran en la tabla 3.

En la tabla 4 se relacionan los antecedentes psiquiátricos relatados por los dolientes, un 40,5\% manifestaba antecedentes de ansiedad y depresión antes de la pérdida, y el $47,2 \%$ tras la misma. Un $35,8 \%$ declararon haber consultado a un facultativo por este motivo, principalmente el médico de atención primaria, en un $68,2 \%$ de los casos. Un 17,4\% necesitó tratamiento farmacológico tras la pérdida. La presencia de duelo prolongado se asoció de manera significativa con haber padecido episodios de ansiedad y/o depresión antes de la pérdida, 61,9\% frente a un
$38,8 \%, p<0,4$ y después de la pérdida, un $90,5 \%$ frente a un 43,9 de aquellos con duelo normal, $p<0,01$. Haber visitado a un psiquiatra se asoció significativamente con la presencia de duelo prologado, un 50\% frente a 14,6\%, $p<0,01$, y también el haber necesitado tratamiento farmacológico para la ansiedad/depresión tras la pérdida, $47,6 \%$ frente a un $15,1 \% \mathrm{p}<0,01$.

En cuanto a la utilización de recursos sanitarios, un 13,7\% visitó el servicio de urgencias hospitalarias tras la pérdida, un $6,4 \%$ precisó ingreso hospitalario, el 58,5\% visito a su médico de atención primaria y a su enfermera, el 17,4\% precisó tratamientos fisioterápicos y el 21,7\% fue derivado por su médico de primaria a otros especialistas. La presencia de duelo prolongado se asoció de manera significativa con un mayor número de visitas a los servicios de urgencias, un 33,3\% frente a al 12,2\%, al médico de atención primaria y a su enfermera, un $90,5 \%$ frente a un $56,1 \%$, y al fisioterapeuta un $42,9 \%$ frente a un $15,5 \%, p<0,01$, como se recoge en la tabla 5 .

\section{Tabla 3. Causas de fallecimiento según la clasificación CIE-10}

\begin{tabular}{lcc}
\hline Causa del fallecimiento & $\mathbf{N}$ & $\mathbf{\%}$ \\
\hline Enfermedades infecciosas y parasitarias & 15 & 5 \\
\hline Neoplasias & 150 & 49,7 \\
\hline $\begin{array}{l}\text { Enfermedades de la sangre, los órganos hematopoyéticos y la inmunidad y otros } \\
\text { trastornos que afectan el mecanismo de la inmunidad }\end{array}$ & 2 & 0,7 \\
\hline Trastornos mentales & 3 & 1 \\
\hline Enfermedades del sistema nervioso y de los sentidos & 15 & 5 \\
\hline Enfermedades del sistema respiratorio & 35 & 11,6 \\
\hline Enfermedades del sistema circulatorio & 47 & 15,6 \\
\hline Enfermedades del sistema digestivo & 3 & 1 \\
\hline Enfermedades de la piel y del tejido subcutáneo & 1 & 0,3 \\
\hline Enfermedades del sistema genitourinario & 5 & 1,7 \\
\hline Malformaciones congénitas & 1 & 0,3 \\
\hline Síntomas y signos no clasificados & 5 & 1,7 \\
\hline Causas externas de mortalidad & 20 & 6,6 \\
\hline Total & 302 & 100 \\
\hline
\end{tabular}


Tabla 4. Antecedentes psiquiátricos de los dolientes según el tipo de duelo medido mediante el cuestionario PG-13

\begin{tabular}{|c|c|c|c|c|c|}
\hline & $\mathbf{N}$ & $\begin{array}{c}\text { Total } \\
(\mathrm{n}=299)\end{array}$ & $\begin{array}{l}\text { Duelo } \\
\text { Normal } \\
(\mathrm{n}=278)\end{array}$ & $\begin{array}{l}\text { Duelo } \\
\text { Prolongado } \\
(\mathbf{n}=21)\end{array}$ & p-valor \\
\hline $\begin{array}{l}\text { Episodios de ansiedad o depresión previos a } \\
\text { la pérdida }(\%)\end{array}$ & 299 & 40,5 & 38,8 & 61,9 & 0,04 \\
\hline $\begin{array}{l}\text { Ha precisado medicación para estos } \\
\text { episodios }(\%)\end{array}$ & 299 & 27,8 & 26,6 & 42,9 & 0,11 \\
\hline $\begin{array}{l}\text { Episodios de ansiedad o depresión tras la } \\
\text { pérdida }(\%)\end{array}$ & 299 & 47,2 & 43,9 & 90,5 & $<0,01$ \\
\hline Ha precisado valoración por facultativo (\%) & 299 & 35,8 & 32 & 85,7 & $<0,01$ \\
\hline $\begin{array}{l}\text { Clase de facultativo }(\%) \\
\text { - Atención primaria } \\
\text { - Psiquiatría } \\
\text { - Psicólogo }\end{array}$ & 107 & $\begin{array}{l}68,2 \\
20,6 \\
50,5\end{array}$ & $\begin{array}{l}68,5 \\
14,6 \\
49,4\end{array}$ & $\begin{array}{c}66,7 \\
50 \\
55,6\end{array}$ & $\begin{array}{c}0,87 \\
<\mathbf{0 , 0 1} \\
0,6\end{array}$ \\
\hline $\begin{array}{l}\text { Ha precisado tratamiento para la ansiedad/ } \\
\text { depresión tras la pérdida }(\%)\end{array}$ & 299 & 17,4 & 15,1 & 47,6 & $<0,01$ \\
\hline $\begin{array}{l}\text { Número de Psicofármacos que toma en la } \\
\text { actualidad Media(DT) }\end{array}$ & 52 & $1,3(0,5)$ & $1,3(0,5)$ & $1,4(0,5)$ & 0,64 \\
\hline
\end{tabular}

Tabla 5. Utilización de recursos sanitarios según la presencia de duelo prolongado medido por el cuestionario PG-13

\begin{tabular}{|c|c|c|c|c|c|}
\hline & $\mathbf{N}$ & $\begin{array}{c}\text { Total } \\
(n=299)\end{array}$ & $\begin{array}{c}\text { Duelo } \\
\text { Normal } \\
(\mathrm{n}=278) \\
\end{array}$ & $\begin{array}{c}\text { Duelo } \\
\text { Prolongado } \\
(\mathrm{n}=21)\end{array}$ & p-valor \\
\hline Ha estado de baja tras la pérdida (\%) & 299 & 8,7 & 9,4 & 0 & 0,23 \\
\hline $\begin{array}{l}\text { Ha visitado el servicio de urgencias tras } \\
\text { la pérdida }(\%)\end{array}$ & 299 & 13,7 & 12,2 & 33,3 & $<0,01$ \\
\hline Número de veces Media(DT) & 41 & $1,3(0,8)$ & $1,3(0,8)$ & $1,2(0,7)$ & 0,9 \\
\hline Ha precisado ingreso en hospital (\%) & 299 & 6,4 & 6,8 & 0 & 0,38 \\
\hline Ha visitado a su MAP $(\%)$ & 299 & 58,5 & 56,1 & 90,5 & $<0,01$ \\
\hline Número de visitas Media(DT) & 175 & $6,0(9,6)$ & $6,0(10,1)$ & $6,05(4,8)$ & 0,9 \\
\hline Ha visitado a su Enfermera (\%) & 299 & 58,5 & 56,1 & 90,5 & $<0,01$ \\
\hline Número de visitas Media(DT) & 96 & $5,3(9,8)$ & $5,5(10,2)$ & $4,2(2,6)$ & 0,7 \\
\hline Ha visitado el servicio de Fisioterapia (\%) & 299 & 17,4 & 15,5 & 42,9 & $<0,01$ \\
\hline Número de visitas Media(DT) & 52 & $7,6(8,2)$ & $7,9(9,3)$ & $6,4(6,3)$ & 0,64 \\
\hline $\begin{array}{l}\text { Ha sido derivado por su MAP a otros } \\
\text { especialistas }(\%)\end{array}$ & 299 & 21,7 & 20,5 & 38,1 & 0,06 \\
\hline Número de derivaciones Media(DT) & 65 & $1,5(0,8)$ & $1,5(0,8)$ & $1,2(0,4)$ & 0,27 \\
\hline Ha recibido nuevos diagnósticos (\%) & 299 & 16,7 & 16,5 & 19 & 0,7 \\
\hline Número de nuevos diagnósticos Media(DT) & 50 & $1,3(0,6)$ & $1,2(0,6)$ & $1,5(1)$ & 0,52 \\
\hline
\end{tabular}


Utilizando los criterios del DSM-IV se diagnosticó trastorno por ansiedad generalizada a un $1,7 \%$ de los dolientes y episodio depresivo mayor a un 9,4\%. Un 6\% presentaron una percepción de apoyo social bajo con el cuestionario Duke. Utilizando el ITRD, el 38,8\% de los dolientes se clasificaron como Duelo Inhibido y el 38,1\% como Duelo Prolongado. El diagnóstico de duelo prolongado con el cuestionario PG-13 se relacionó de manera significativa con la presencia de trastorno por ansiedad generalizada, episodio depresivo mayor, un apoyo social percibido bajo y Duelo Prolongado según los criterios del ITRD, p<0,01. Tabla 6.

Se ajustó un modelo multivariante para buscar factores asociados a duelo prolongado. Se utilizaron las variables asociadas a duelo prolongado en el análisis univariable como variables independientes y la presencia de duelo prolongado como variable dependiente. El haber consultado a un psiquiatra tras la pérdida $(\mathrm{OR}=5,331$, $p=0,01)$, tener unos ingresos mensuales menores a 2000 euros $(\mathrm{OR}=4,609$, $\mathrm{p}=0,042)$ y cumplir criterios de episodio depresivo mayor $(\mathrm{OR}=7,567, \mathrm{p}=0,001)$ se asociaron con la presencia de duelo prolongado. El 36\% de la varianza del modelo se explicó por estos factores. Tabla 7.

\section{DISCUSIÓN}

La prevalencia de duelo prolongado encontrada en este trabajo, 7,023\% (IC95\%: 4,4-10,05), es similar a la de otros estudios, un $6,7 \%$ de duelo prolongado en dolientes en Alemania y Países bajos ${ }^{(22,23)}$ y un $9 \%$ en Estados Unidos ${ }^{(1)}$.

De los 329 dolientes a los que se ofreció participar en este estudio, aceptó un $93,1 \%$, una cifra elevada. El hecho de que sea su propio médico o enfermera de atención primaria o los mismos profesionales de cuidados paliativos que atendieron al fallecido y a su familia en la Unidad de

Tabla 6. Presencia de Trastorno por ansiedad generalizada, Episodio depresivo mayor, apoyo social percibido y clasificación del Inventario Texas revisado de Duelo según la presencia o no de duelo prolongado medido por el cuestionario PG-13

\begin{tabular}{|c|c|c|c|c|}
\hline & $\begin{array}{c}\text { Total } \\
(\mathrm{n}=299)\end{array}$ & $\begin{array}{c}\text { Duelo } \\
\text { Normal } \\
(n=278)\end{array}$ & $\begin{array}{c}\text { Duelo } \\
\text { Prolongado } \\
(n=21)\end{array}$ & p-valor \\
\hline Trastorno por Ansiedad Generalizada (\%) & 1,7 & 0,4 & 19 & $<0,01$ \\
\hline Episodio Depresivo Mayor (\%) & 9,4 & 6,5 & 47,6 & $<0,01$ \\
\hline $\begin{array}{l}\text { Apoyo social percibido Bajo. Cuestionario } \\
\text { Duke }(\%)\end{array}$ & 6 & 4,3 & 28,6 & $<0,01$ \\
\hline \multicolumn{5}{|l|}{ Inventario Texas Revisado sobre Duelo (\%): } \\
\hline - Duelo Resuelto & 9,7 & 10,4 & 0 & 0,24 \\
\hline - Duelo Inhibido & 38,8 & 41,7 & 0 & $<0,01$ \\
\hline - Duelo Retrasado & 13,4 & 14,4 & 0 & 0,89 \\
\hline - Duelo Prolongado & 38,1 & 33,5 & 100 & $<0,01$ \\
\hline
\end{tabular}




\section{Tabla 7. Análisis multivariable de los factores de riesgo asociados a duelo prolongado $(n=107)$}

\begin{tabular}{lcccccc}
\hline Variables predictoras & $\mathbf{B}$ & $\begin{array}{c}\text { Error } \\
\text { estándar }\end{array}$ & Wald & $\mathbf{p}$ & $\begin{array}{c}\text { Odds } \\
\text { Ratio }\end{array}$ & $\begin{array}{c}\text { IC95\% para } \\
\text { Odds Ratio }\end{array}$ \\
\hline Valoración por psiquiatra & 1,673 & 0,652 & 6,589 & 0,010 & 5,331 & $1,485-19,131$ \\
\hline $\begin{array}{l}\text { Ingresos mensuales } \\
\text { menores de 2000€ }\end{array}$ & 1,528 & 0,752 & 4,125 & 0,042 & 4,609 & $1,055-20,137$ \\
\hline Episodio depresivo mayor & 2,024 & 0,635 & 10,148 & 0,001 & 7,567 & $2,178-26,281$ \\
\hline
\end{tabular}

Cuidados Paliativos o en su propio domicilio, crea una base de confianza al doliente y favorece su participación ${ }^{(24)}$, pero también sabemos que precisamente por ser el profesional que le atiende habitualmente o el que le atendió en momentos muy críticos el que se lo pida, es más difícil que digan que no por miedo a que repercuta negativamente en la relación futura médico-paciente, aunque se les asegure por escrito que no va a ser asi ${ }^{(25)}$.

Para los dolientes, expresar sus sentimientos durante la entrevista pudo ser doloroso, pero en la mayor parte de los casos agradecieron tener la oportunidad de hablar de ellos abiertamente con alguien distinto de sus familiares y allegados. Esto coincide con el estudio de Seamark, et al., ${ }^{(26)}$ realizado para recoger las opiniones de los familiares de pacientes fallecidos en hospitales de cuidados paliativos acerca del servicio recibido en el mismo, en el que un $83 \%$ de los dolientes entrevistados no presentaron malestar durante la entrevista o este fue leve y el $75 \%$ encontraron la entrevista útil o muy útil.

A todos los participantes se les ofreció ayuda psicológica gratuita en caso de que quisieran utilizarla. A aquellos que parecían más afectados y/o con menos recursos personales de afrontamiento se les recomendó utilizarla, siguiendo la Guía de comportamiento ético en investigación con dolientes de Parkes ${ }^{(21)}$.

En los estudios con dolientes es difícil encontrar datos sobre los pacientes que no desean participar, aunque se sabe que el rechazo a la participación se relaciona con peores procesos de duelo. En el estudio de Stiel et al., ${ }^{(27)}$ un $16,2 \%$ de los dolientes invitados a participar rechazaron hacerlo. Entre los motivos recogidos se encontraban sentirse sobrecargados emocionalmente, no querer pensar sobre la muerte de su ser querido o que no había pasado tiempo suficiente para hablar de ello. En nuestro estudio uno de cada cuatro dolientes que rechazó participar adujo que no tenía tiempo, un $17,4 \%$ estar muy triste y un $7 \%$ no querer recordar.

Del grupo que rechazó participar tenemos algunas variables significativas, como que viven con un número menor de personas y que el fallecido fue mayoritariamente varón, y otras no significativas pero que nos ilustran la tendencia de este grupo, como que el fallecido era familiar de primer grado en el $100 \%$ de los casos y el doliente mujer en el $81 \%$ de los casos. Nos encontramos ante un colectivo vulnerable, mujeres que viven solas o con otra persona y que han perdido a un familiar muy cercano, padre, hijo o esposo, y que además presentan dificultades para hablar de su sufrimiento.

Entre aquellos que participaron encontramos en el análisis univariante que la presencia de duelo prolongado se asoció de manera significativa con la edad media del fallecido, 71,6 (DT=17,1) años en el grupo de duelo normal frente a los 60,6 (DT=21,8) años en el grupo de duelo prolongado. Las 
pérdidas de gente joven o en la infancia, han demostrado ser un factor importante en la mala evolución del duelo, ya que cuanto menor es la edad del fallecido más difícil es aceptar la pérdida ${ }^{(6,7)}$.

Las causas de la muerte fueron codificadas mediante la clasificación CIE-10 con la intención de ser lo mas exhaustivos posibles, pero a la hora de analizar encontramos que incluso agrupando estas causas en los 12 epígrafes principales e intentado relacionarlos con la presencia de duelo prolongado, el $75 \%$ de las casillas estaban vacías, lo que hizo imposible cualquier análisis estadístico.

La presencia en el fallecido de una enfermedad susceptible de recibir cuidados paliativos se asoció significativamente con un duelo normal como aparece en el estudio de Bonnano et al., ${ }^{(28)}$ en el que la presencia de los patrones duelo normal o depresión-mejoría se asociaron a haber tenido un cónyuge enfermo antes de fallecer y el patrón duelo crónico se asoció en su estudio con haber tenido una pareja sana antes de fallecer.

El fallecido tenía una enfermedad susceptible de recibir cuidados paliativos en el $74,1 \%$ de los casos con duelo normal frente a un $52,4 \%$ de los dolientes con duelo prolongado, $p<0,03$. La necesidad de cuidados paliativos se asocia con enfermedades de larga evolución, que permiten tomar conciencia del desenlace con anterioridad, anticipar el duelo y esto favorece el reajuste tras la muerte ${ }^{(29-31)}$. Este es el trabajo del ESADP. En los casos en los que la asistencia domiciliaria es prolongada, el trabajo de médicos y enfermeras con las familias también ayuda a éstas a tomar conciencia y perspectiva de la situación y a adelantar lo que va a venir. Además tienen la capacidad de derivar al psicólogo del equipo a aquellos pacientes o familiares que presentan más dificultades para afrontar la situación favoreciendo el reajuste posterior ${ }^{(32,33)}$.
En la variable centro de captación se encontraron diferencias estadísticamente significativas entre los pacientes captados en los hospitales de cuidados paliativos y los captados en el ESADP, $p=0,001$. Los psicólogos de hospitales de cuidados paliativos recogieron al $26,1 \%$ de los dolientes que participaron en el estudio, sin embargo entre ellos se encuentra el 52,4\% de los dolientes con duelo prolongado. Esto se debe al funcionamiento interno de las Unidades de Cuidados Paliativos de los Hospitales de Apoyo, ya que reciben asistencia psicológica aquellos familiares de enfermos que los profesionales derivan a su servicio cuando detectan que tienen múltiples factores de riesgo para una mala evolución del duelo o que la familia o el paciente están sobrepasados por la situación. De esta manera, la muestra atendida por los psicólogos presenta un sesgo de selección. En atención primaria, sin embargo, se invitó a participar a los familiares de los pacientes de los que se tenía conocimiento de su fallecimiento y a todo aquel que manifestó haber sufrido una pérdida en los plazos establecidos para el estudio, sin otro filtro.

El $54,8 \%$ de los participantes se recogieron a partir de los casos atendidos en el ESADP, entre los cuales la presencia de duelo prolongado representó un $19 \%$ del total de casos. Los dolientes del ESADP fueron captados consecutivamente a partir de los listados de defunciones del equipo, sin otro criterio. La baja representación de duelo prolongado entre los dolientes recogidos en el ESADP probablemente se relaciona con lo expuesto anteriormente. Trabajan esencialmente con pacientes con enfermedad crónica avanzada oncológica o por insuficiencia crónica de órgano, habitualmente la mediana del tiempo se seguimiento del paciente es de 4-6 semanas y se atienden tanto las necesidades del paciente como de su familia preparando a ambos para 
el desenlace inevitable. De esta manera cuando la muerte llega, están un poco más preparados.

La presencia de duelo prolongado se asoció en el análisis univariante con ser mujer como se recoge en la literatura ${ }^{(34,35)}$. Sin embargo, en nuestro estudio no se encontró significación estadística en cuanto a edad, parentesco, tiempo transcurrido desde el fallecimiento, número de hijos, nivel educativo, profesión, número de adultos y número de menores en el domicilio, que son otros factores conocidos de riesgo de aparición de duelo prolongado.

La presencia de duelo prolongado se asoció con la situación laboral del doliente. La variable tenía siete categorías cuando se recogió: activo y trabaja, activo y no trabaja, incapacidad temporal, incapacidad permanente, desempleado con prestación, desempleado sin prestación, ama de casa, jubilado y estudiante. Se decidió agruparla en una variable dicotómica, trabaja o no trabaja, para su estudio. Se encontró que de aquellos con duelo normal, trabajaban el $51,4 \%$ frente a un $48,6 \%$ que no lo hacía, sin embargo entre los que presentaban duelo prolongado trabajaba tan solo un $23,8 \%$ frente a un $76,2 \%$ que no, $p=0,015$. El trabajo aporta varias cosas positivas, aparte de la remuneración monetaria, reconocimiento de la labor realizada, una red social, un motivo para salir de casa y vertebra de cierta manera la rutina diaria ${ }^{1}$. El desempleo, ser ama de casa o jubilado pueden favorecer cierto grado de soledad, dependencia económica y frustración personal. Este dato de empleo en nuestra muestra se relaciona con la situación económica.

La variable ingresos se recogió como categórica, en intervalos expresados en euros, pero para el análisis se decidió convertirla en numérica, usando el promedio de los intervalos para poder calcular las medias y sus desviaciones estándar. El salario medio en la Comunidad de Ma- drid en el Año 2013 según el INE fue de $26.215,36$ euros, unos 2.184,61 euros al mes, pero no hemos encontrado el dato en el INE de los ingresos mensuales medios familiares $^{(36)}$. En un estudio realizado por una consultora independiente ${ }^{(37)}$ se recogieron los ingresos familiares medios mensuales, que para Madrid capital eran unos 2.110 euros al mes. En nuestra muestra, los ingresos mensuales medios fueron de 2.149,03 euros, 2.211,39 en el caso de los que tenían duelo normal. Sin embargo, aquellos que presentaban duelo prolongado tuvieron de media unos ingresos familiares de 1.436,8 euros al mes, siendo las dificultades económicas un factor conocido de mala evolución del duelo ${ }^{(38,39)}$.

La presencia de duelo prolongado también se asoció de manera significativa en el análisis univariante con la presencia de trastorno por ansiedad generalizada, episodio depresivo mayor, apoyo social percibido bajo y la presencia de duelo prolongado medido con el cuestionario Inventario Texas Revisado de Duelo. La relación entre la presencia de duelo prolongado, ansiedad generalizada y episodio depresivo mayor replica lo aparecido en otros estudios en los que la presencia de otras comorbilidades psiquiátricas se asocia a una peor evolución del duelo $\mathrm{o}^{(5,14,38,40-42)}$.

En relación con la presencia de duelo prolongado y el uso de servicios sanitarios los resultados son congruentes con estudios previos. Así en el estudio de Charlton et al., ${ }^{(43)}$ se encontró que la frecuentación al médico de familia paso de una media de 5,99 visitas al año en el año previo a enviudar a 7,60 visitas el año posterior, la diferencia de 1,61 (IC95\%: 0,398-2,822) fue estadísticamente significativa. Al ser nuestro estudio transversal, no disponemos de datos de frecuentación médica antes y después de la pérdida. En términos globales el facultativo más visitado fue el médico de familia, en el 68,2\% de los casos, seguido del psicólogo en 
un $50,5 \%$, y finalmente del psiquiatra en el $20,6 \%$ de los casos. La presencia de duelo prolongado se asoció en el análisis univariante únicamente a haber visitado a un psiquiatra tras la pérdida, un 50\% de los que presentaron duelo prolongado visitaron al psiquiatra frente al $14,6 \%$ de aquellos con duelo normal. De manera similar, en el estudio de Lichtental et al., ${ }^{(41)}$ el $40 \%$ de los dolientes con duelo prolongado había utilizado un servicio de salud mental tras la pérdida y el $63 \%$ de aquellos sin duelo prolongado pero con otra comorbilidad psiquiátrica como episodio depresivo mayor o trastorno por ansiedad generalizada. También aquellos con duelo prolongado visitaron más el servicio de urgencias, a su médico y a su enfermera de atención primaria y el servicio de fisioterapia de manera estadísticamente significativa, estos resultados se corresponden con los aparecidos en la literatura. No se encontraron diferencias entre los dolientes con duelo normal y prolongado entre los ingresos en el hospital, si precisó baja laboral, si ha precisado derivación a otros especialistas ni en el número de visitas a cada profesional.

En el estudio de Shah et al., ${ }^{(44)}$ el riesgo relativo de recibir medicación psicotrópica fue de media un 9,5\% (IC95\% 9,1-9,9) a los 2 meses tras la pérdida y un $17,9 \%$ (IC95\% 17,3-18,4) a los 12 meses. De manera similar en este estudio, el $17,4 \%$ de los dolientes tomaba algún psicofármaco en el momento del estudio, el 47,6\% de los dolientes con duelo prolongado frente al $15,1 \%$ de los dolientes con duelo normal. La cifra de pacientes que toman medicación entre aquellos con duelo normal, parece adecuada ya que se engloban en esta cifra tanto antidepresivos como benzodiazepinas, pero entre aquellos con duelo prolongado, dada la elevada tasa de comorbilidad, el hecho de que el $50 \%$ hayan consultado a un psiquiatra es positivo, pero habría que estudiar por qué la otra mitad no recibe ningún tipo de terapia ni tratamiento farmacológico. En nuestro estudio no se encontraron diferencias en cuanto al número de fármacos que tomaban en cada grupo.

En el análisis multivariable de los factores de riesgo asociados a duelo prolongado encontramos que tres variables explicaron el 36\% del modelo: cumplir criterios de episodio depresivo mayor, haber precisado valoración psiquiátrica tras la pérdida y unos ingresos mensuales inferiores a 2.000 euros. Ya hemos señalado que aquellos dolientes con duelo prolongado y otra patología psiquiátrica presentan peor evolución del duelo y es más probable que soliciten ayuda profesional ${ }^{(41)}$. Sin embargo, la cifra de 2.000 euros mensuales por hogar puede parecer un poco elevada en comparación con los salarios medios españoles, esto puede estar en relación con que la comunidad de Madrid se encuentra entre las que mayores salarios medios tiene de España ${ }^{(36)}$, que se recogieron muchos dolientes en el barrio de Chamberí de Madrid, que está entre los de renta más alta de la ciudad, y que en esta muestra hay un $60,9 \%$ de profesionales cualificados, un 52,4\% entre aquellos con duelo prolongado.

En resumen podemos afirmar que la prevalencia de duelo prolongado encontrada es de 7,023\%, similar a estudios previos en países occidentales y que la presencia del mismo se asoció con tener unos ingresos menores de 2.000 euros al mes, haber precisado derivación a psiquiatría y cumplir criterios de episodio depresivo mayor en el momento de la entrevista. Las personas con duelo prolongado han hecho una mayor utilización de los servicios sanitarios, tanto de las consultas del médico de familia como de la enfermera de atención primaria, los servicios de fisioterapia y el servicio de urgencias hospitalarias en mayor proporción que las personas con duelo normal. La presencia de duelo prolongado 
se asoció a la presencia de trastorno por ansiedad generalizada, episodio depresivo mayor y duelo prolongado, así como a un apoyo social percibido más bajo que aqueIlos con duelo normal.

Para finalizar, el duelo prolongado es un cuadro que aumenta el riesgo de sufrir trastornos en la salud física y mental, se asocia a otras enfermedades mentales graves y genera un aumento en la utilización de recursos sanitarios. Es, por tanto, una entidad a tener en cuenta en la práctica clínica diaria ya que puede ser el motivo oculto tras muchas consultas médicas. Detectarlo a tiempo y derivarlo a recursos específicos como psicoterapia o grupos de dolientes para su tratamiento le ahorrará a los dolientes tiempo y sufrimiento.

\section{REFERENCIAS BIBLIOGRÁFICAS}

1. Prigerson HG, Vanderwerker LC, Maciejewski PK. A case for inclusion of prolonged grief disorder in DSM-V. En: Stroebe MS, Hansson RO, Schut $H$, Stroebe W. Handbook of bereavement research and practice: 21st Century Perspectives. Washington DC, EEUU: American Psychological Association; 2008. p.165-86.

2. Prigerson HG, Bierhals AJ, Kasl SV, Reynolds CF, Shear MK, Day N, et al. Traumatic grief as a risk factor for mental and physical morbidity. Am J Psychiatry 1997;154:616-23. Doi: 10.1176/ ajp.154.5.616

3. Elwert F, Christakis NA. The effect of widowhood on mortality by the causes of death of both spouses. Am J Public Health 2008;98:2092-8. Doi: 10.2105/ AJPH.2007.114348

4. Pitman A, Osborn D, King M, Erlangsen A. Effects of suicide bereavement on mental health and suicide risk. Lancet Psychiatry 2014;1:86-94. Doi: 10.1016/S22150366(14)70224-X

5. Prigerson HG, Horowitz MJ, Jacobs SC, Parkes CM, Aslan M, Goodkin K, et al. Pro- longed Grief Disorder: Psychometric Validation of Criteria Proposed for DSM-V and ICD-11. PLoS Med [edición electrónica]. 2009 [Acceso el 21/03/2015].Disponible en: http://journals.plos.org/plosmedicine/ article?id=10.1371/journal.pmed.1000121

6. Bonnano GA, Kaltman S. The varieties of grief experience. Clin Psychol Rev 2001;5;705-734. Doi:10.1016/S02727358(00)00062-3

7. Rosner R. Prolonged grief: setting the research agenda. Eur J Psychotraumatol 2015;6:27303. Doi: 10.3402/ejpt. v6.27303.

8. Wittouck C, Van Autreve S, De Jaegere E, Portzky G, van Heeringen K. The prevention and treatment of complicated grief: A meta-analysis. Clin Psychol Rev 2011;31:69-78. Doi: 10.1016/j. cpr.2010.09.005.

9. Hensley PL, Slonimski CK, Uhlenhuth EH, Clayton PJ. Escitalopram: an open-label study of bereavement-related depression and grief. J Affect Disord 2009;113:142149. Doi: 10.1016/j.jad.2008.05.016.

10. Simon NM, Shear MK, Fagiolini A, Frank E, Zalta A, Thompson EH, et al. Impact of concurrent naturalistic pharmacotherapy on psychotherapy of complicated grief. Psychiatry Res 2008;159:31-6. Doi: 10.1016/j.psychres.2007.05.011

11. Reynolds CF, Miller MD, Pasternak RE, Frank E, Perel JM, Cornes C, et al. Treatment of bereavement-related major depressive episodes in later life: a controlled study of acute and continuation treatment with nortriptyline and interpersonal psychotherapy. Am J Psychiatry 1999;156:202-8.

12. Olmeda García MS, García Olmos A, Basurte Villamar I. Rasgos de personalidad en duelo complicado. En: Interpsiquis. 3er congreso virtual de Psiquiatría. Psiquiatría. com;2002. [actualizado el 6 de febrero de 2002, [acceso 25 de diciembre de 2010]. Disponible en: http://www.psiquiatria. com/articulos/depresion/5372/. 
13. Xiao-Lin H, Xiao-Lin L, Xin-Man D, Rong L. Factors related to complicated grief among bereaved individuals after the Wenchuan earthquake in China. Chin Med J 2015; 128:1438-43. Doi: 10.4103/03666999.157647

14. Simon NM, Shear KM, Thompson EH, Zalta AK, Perlman C, Reynolds CF, et al. The prevalence and correlates of psychiatric comorbidity in individuals with complicated grief. Compr Psychiatry 2007;48:395-9. Doi: 10.1016/j.comppsych.2007.05.002

15. Simon NM, Pollack MH, Fischmann D, Perlman CA, Muriel AC, Moore CW, et al. Complicated grief and its correlates in patients with bipolar disorder. J Clin Psychiatry 2005;66:1105 -10.

16. Shear K, Frank E, Houck PR, Reynolds CF 3rd. Treatment of complicated grief: A randomized controlled trial. JAMA 2005;293:2601-8. Doi:10.1001/ jama.293.21.2601

17. Faschingbauer TR, Devaul RA, Zisook S. Development of the Texas Inventory of Grief. Am J Psychiatry 1977;134:696-8. Doi:10.1176/ajp.134.6.696

18. García García JA, Landa Petralanda V, Trigueros Manzano MC, Gaminde Inda I. Inventario Texas Revisado de Duelo (ITRD): adaptación al castellano, fiabilidad y validez. Aten Primaria 2005;35:353-8.

19. Bellón Saameño JA, Delgado Sánchez A, Luna del Castillo JD, Lardelli Claret P. Validez y fiabilidad del cuestionario de apoyo social funcional Duke-UNC-11. Aten Prim 1996;18;153-63.

20. Patricia Estevan Burdeus. Validación y adaptación trascultural del cuestionario Prolonged grief PG-13 para la detección precoz de duelo prolongado.[tesis doctoral]. Madrid: Universidad Complutense de Madrid: 2015.

21. Parkes CM. Guidelines for conducting ethical bereavement research. Death Stud 1995;19:171-81. Doi:10.1080/07481189508252723

22. Newson RS, Boelen PA, Hek K, Hofman $A$, Tiemeier $H$. The prevalence and cha- racteristics of complicated grief in older adults. J Affect Disord 2011;132:231-8. Doi: 10.1016/j.jad.2011.02.021

23. Kersting A, Brähler E, Glaesmer H, Wagner B. Prevalence of complicated grief in a representative population based sample. J Affect. Disord 2011;131:339-43. Doi: 10.1016/j.jad.2010.11.032

24. Gysels M, Shipman C, Higginson IJ. «I will do it if it will help others»: Motivations among patients taking part in qualitative studies in palliative care. J Pain Symptom Manage 2008;35:347-55. Doi: 10.1016/j.jpainsymman.2007.05.012

25. Cook AS. The dynamics of ethical decision making in bereavement research. En: Stroebe MS, Hansson RO, Stroebe W, Schut $\mathrm{H}$. Handbook of bereavement research: Consequences, coping, and care. Washington DC, EEUU: American Psychological Association; 2001.p.119-142.

26. Seamark DA, Gilbert J, Lawrence CJ, WiIliams S. Are postbereavement research interviews distressing to carers? Lessons learned from palliative care research. PaIliat Med 2000;14:55-6.

27. Stiel S, Heckel M, Bussmann S, Weber M, Ostgathe C. End-of-life care research with bereaved informal caregivers--analysis of recruitment strategy and participation rate from a multi-centre validation study. BMC Palliat Care 2015;14:21. Doi: 10.1186/s12904-015-0020-4.

28. Bonanno GA, Wortman CB, Lehman DR, Tweed RG, Haring M, Sonnega J, et al. Resilience to loss and chronic grief: A prospective study from preloss to 18-months postloss. J Pers Soc Psychol 2002;83:1150-64. Doi:10.1037/00223514.83.5.1150

29. Barry LC, Kasl SV, Prigerson HG. Psychiatric disorders among bereaved persons: the role of perceived circumstances of death and preparedness for death. Am J Geriatr Psychiatry 2002;10:447-57. Doi:10.1097/00019442-20020700000011 
30. Hebert RS, Dang Q, Schulz R. Preparedness for the death of a loved one and mental health in bereaved caregivers of patients with dementia: Findings from the REACH study. J Palliat Med 2006;9:68393. Doi:10.1089/jpm.2006.9.683.

31. Bradley EH, Prigerson H, Carlson MDA, Cherlin E, Johnson-Hurzeler R, KasI SV. Depression among surviving caregivers: does length of hospice enrollment matter? Am J Psychiatry 2004;161:2257-62. Doi:10.1176/appi.ajp.161.12.2257

32. Lacasta Reverte MA, Limonero García JT, García Penas A, Barahona H. La atención al duelo en cuidados paliativos. Análisis de los servicios prestados en España. Med Paliat 2014. Doi:10.1016/j.medipa.2014.05.006

33. Yi P, Barreto P, Soler C, Fombuena $M$, Espinar V, Pascual L, et al. Grief support provided to caregivers of paIliative care patients in Spain. PaIliat Med 2006;20:521-31. Doi: 10.1191/0269216306pm1165oa

34. Agnew A, Manktelow R, Taylor B, Jones L. Bereavement needs assessment in specialist palliative care: A review of the literature. Palliat Med 2010;24:46-59. Doi: 10.1177/0269216309107013.

35. Fujisawa D, Miyashita M, Nakajima S, Ito M, Kato M, Kim Y. Prevalence and determinants of complicated grief in general population. J Affect Disord 2010:127;352-

8. Doi: 10.1016/j.jad.2010.06.008

36. INE. Encuesta Anual de Estructura Salarial. Año 2013. [monografía en Internet]. Madrid: Instituto Nacional de Estadistica; 2015 [acceso 28/09/2015]. Disponible en: www.ine.es/prensa/np916.pdf

37. AIS Group [sede Web]*. Barcelona: AIS Aplicaciones de Inteligencia Artificial, S.A. 2015 [acceso 28 de septiembre de 2015]. Los hogares españoles ingresaron en 2014 una media de $1.880 €$ mensuales. Dispo- nible en: http://www.ais-int.com/los-hogaresespanoles-ingresaron-en-2014-unamedia-de-1-880e-mensuales.html"

38. Barreto P, Torre O, Pérez-Martín M. Detección de duelo complicado. Psicooncología 2012; 9:355-68. Doi: 10.5209/ rev_PSIC.2013.v9.n2-3.40902

39. Goldsmith B, Morrison RS, Vanderwerker LC, Prigerson HG. Elevated rates of prolonged grief disorder in African Americans. Death Stud 2008;32:352-65. Doi: 10.1080/07481180801929012.

40. Robinaugh DJ, McNally RJ, LeBlanc NJ, Pentel KZ, Schwarz NR, Shah RM, et al. Anxiety sensitivity in bereaved adults with and without complicated grief. J Nerv Ment Dis 2014;202:620-2. Doi: 10.1097/ NMD.0000000000000171.

41. Lichtenthal WG, Nilsson M, Kissane DW, Breitbart W, Kacel E, Jones EC, et al. Underutilization of mental health services among bereaved caregivers with prolonged grief disorder. Psychiatr Serv 2011;62:1225-9. Doi: 10.1176/appi. ps.62.10.1225.

42. Vanderwerker LC, Laff RE, Kadan-Lottick NS, McColl S, Prigerson HG. Psychiatric disorders and mental health service use among caregivers of advanced cancer patients. J Clin Oncol 2005;23:6899907. Doi: 10.1200/JCO.2005.01.370

43. Charlton R, Sheahan K, Smith G, Campbell I. Spousal bereavement-implications for health. Fam Pract 2001;18:614-8. Doi: 10.1093/fampra/18.6.614

44. Shah SM, Carey IM, Harris T, DeWilde S, Victor CR, Cook DG. Initiation of psychotropic medication after partner bereavement: a matched cohort study. PLoS ONE. PLoS Med [edición electrónica]. 2013 [consultado el 21/10/2015]. Disponible en: http://journals.plos.org/ plosone/article?id=10.1371/journal. pone.0077734. 
